\title{
Mediterranean Diet and Physical Activity Protect from Silent Brain Infarcts in a Cohort of Patients with Atrial Fibrillation
}

\author{
Irene Escudero-Martínez, ${ }^{\mathrm{a}, \mathrm{b}}$ Fernando Mancha, ${ }^{\mathrm{b}}$ Ángela Vega-Salvatierra, ${ }^{\mathrm{b}}$ María Irene Ayuso, ${ }^{\mathrm{b}}$ \\ Rafael F. Ocete, ${ }^{\mathrm{c}}$ Pilar Algaba, ${ }^{\mathrm{b}}$ Antonio López-Rueda, ${ }^{\mathrm{d}}$ Pilar Piñero, ${ }^{\mathrm{c}}$ Elena Fajardo, ${ }^{\mathrm{c}}$ \\ José Román Fernández-Engo, ${ }^{\mathrm{e}}$ Eva María Martín-Sánchez, ${ }^{\mathrm{f}}$ Alejandro Galvao-Carmona, ${ }^{\mathrm{g}}$

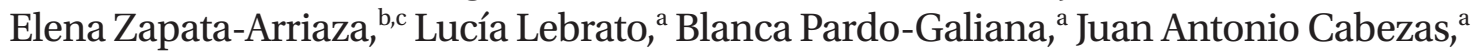 \\ Alejandro González, ${ }^{\mathrm{b}, \mathrm{c}}$ Francisco Moniche, ${ }^{\mathrm{a}, \mathrm{b}}$ Joan Montaner ${ }^{\mathrm{b}, \mathrm{h}}$ \\ aDepartment of Neurology, University Hospital Virgen del Rocío, Sevilla, Spain \\ ${ }^{b}$ Neurovascular Research Laboratory, Institute of Biomedicine of Seville-IBIS, Sevilla, Spain \\ 'Department of Radiology, University Hospital Virgen del Rocío, Sevilla, Spain \\ 'Department of Radiology, Hospital Clínic, Barcelona, Spain \\ IT Department, Andalusian Healthcare Ministry, Sevilla, Spain \\ fInformation Systems Coordination Service, Support Services of the Andalusian Health Service, Seville, Spain \\ ${ }^{9}$ Department of Psychology, Loyola Andalucia University, Sevilla, Spain \\ hDepartment of Neurology, University Hospital Virgen Macarena, Sevilla, Spain
}

\section{Dear Sir:}

Several studies have demonstrated that Mediterranean diet $(\mathrm{MeD})$ has beneficial effects in the prevention of cardiovascular disease (CVD) ${ }^{1,2}$ globally but also in stroke prevention., ${ }^{3,4}$ However, there are few data of the influence of MeD in silent brain infarcts (SBI). Physical activity is also a well-known protective factor for CVD and stroke. ${ }^{5}$ Atrial fibrillation (AF) increases the risk of overt stroke and also SBI. We hypothesize that a healthy lifestyle would reduce the rate of SBI among AF patients. The aim of this study is to determine the association of a healthy profile (the combination of a good MeD adherence plus high level of physical activity) and the prevalence of SBI in a population with AF.

To determine this association we have selected patients diagnosed of non-valvular AF (NVAF) from Seville urban area were selected from DIRAYA, the Andalusian electronic healthcare database. The inclusion criteria were: patients diagnosed of NVAF (according to International Classification of Diseases, 9th revision [ICD-9] classification) who scored 0-1 in the $\mathrm{CHADS}_{2}$ score, age $\geq 50$ years and patients who give their consent. Patients were excluded when they had history of previous stroke/transient ischemic attack, if brain magnetic resonance imagine (MRI) was contraindicated or claustrophobia or when receiving oral anticoagulation. A total of 915 eligible patients were selected. A phone-call was made to explain the study protocol. After inclusion, patients were scheduled for a brain 3T-MRI. Baseline visit was performed at the same time and demographical data and medical history were collected. Physical activity and diet habits were also assessed by two validated self-administered questionnaires, the International Physical Activity Questionnaire (IPAQ) ${ }^{6}$ and the 14-item questionnaire of adherence to MeD from the Prevención con dieta mediterránea (PREDIMED) Study. ${ }^{2}$ All MRI scans were performed with the same 3.0 Tesla MR (Philips Ingenia CX, Release 3.1, Koninklijke, Amsterdam, the Netherlands). MRI protocol included: sagittal $1 \mathrm{~mm}$ 3DT1weighted gradient echo (GRE), sagittal 1,12 mm 3DT2-weighted fat sat fluid-attenuated inversion recovery (FLAIR), axial $5 \mathrm{~mm}$ 2DT2*-weighted GRE, and axial $3 \mathrm{~mm} 2 \mathrm{D}$ diffusion-weighted imaging (DWI [B0, B1000, ADC map]). SBI were defined as focal lesions with roughly the same intensity of cerebrospinal fluid. SBI were classified into acute/subacute lesions (hyperintense 
Table 1. Multivariable analysis of silent brain infarct risk factors

\begin{tabular}{lccc}
\hline Variable & $P$ & Odds ratio & 95\% Confidence interval \\
\hline Age $>62.5 \mathrm{yr}$ & 0.000 & 3.47 & $1.72-6.97$ \\
Healthy profile & 0.046 & 0.49 & $0.24-0.99$ \\
Hypertension & 0.655 & 0.88 & $0.51-1.53$ \\
Diabetes & 0.878 & 1.09 & $0.37-3.20$ \\
Hyperlipidemia & 0.633 & 1.14 & $0.66-1.98$ \\
Alcohol & 0.160 & 2.00 & $0.76-5.26$ \\
Tobacco & 0.196 & 1.65 & $0.77-3.51$
\end{tabular}

signal lesion on DWI) and chronic lesions (hyperintense FLAIR signal lesions with hypointense signal on T1-weigted images). In doubtful cases, we also analyzed the presence of complete surrounding FLAIR hyperintensities as another criterion following the standards for reporting vascular changes in neuroimaging (STRIVE) guidelines. ${ }^{?}$

Statistical analysis was performed with the SPSS version 21.0 statistical package (IBM Co., Armonk, NY, USA). Cutoff values for age and alcohol intake were determined using receiver operating characteristic (ROC) analysis. To determine the independent risk factors of $\mathrm{SBI}$, multivariable regression analysis was performed. Results are shown as odds ratio (OR), 95\% confidence intervals (Cls), and $P$-values. The study protocol and consent forms were approved by the Ethics Committee of Virgen del Rocío University Hospital (reference no.2014PI/162-1).

Of 443 patients included from May 2015 to June 2016, 66 patients (14.9\%) presented at least 1 SBI on MRI. Baseline characteristics are shown in Supplementary Table 1.

Univariate analysis (Supplementary Table 2) showed that an older age ( 71 years old vs. 65 years old, $P=0.001$ ), and higher alcohol intake, defined by consumption of $>78 \mathrm{~g} /$ week (36.4\% vs. $22.3 \%, P=0.014$ ) were associated with higher risk of $\mathrm{SBI}$. There was no association of SBI with MeD adherence nor physical activity when considered individually. Patients with a high adherence to MeD were more physically active (40.6\% vs. $34.6 \%, P=0.028$ ) (Supplementary Figure 1). A variable that combines a good adherence to MeD (score 8-14 in the questionnaire $)^{8}$ plus high level of physical activity $(\geq 3,000$ metabolic equivalents [METS]/week) was created (healthy profile). In univariate analysis healthy profile was associated with a lower risk of SBI ( $16.6 \%$ vs. $30 \%, P=0.035)$. After logistic regression analysis adjusted by vascular risk factors (Table 1 ), age $\geq 62.5$ years was an independent predictor of $\mathrm{SBI}(\mathrm{OR}, 3.47 ; 95 \% \mathrm{Cl}$, 1.72 to $6.97 ; P<0.001)$ and a healthy profile was independently associated with a lower risk of $\mathrm{SBI}(\mathrm{OR}, 0.49 ; 95 \% \mathrm{Cl}, 0.24$ to 0.98; $P=0.046$ ). Patients $\geq 62.5$ years with low adherence to MeD plus low/moderate level of physical activity had high fre-

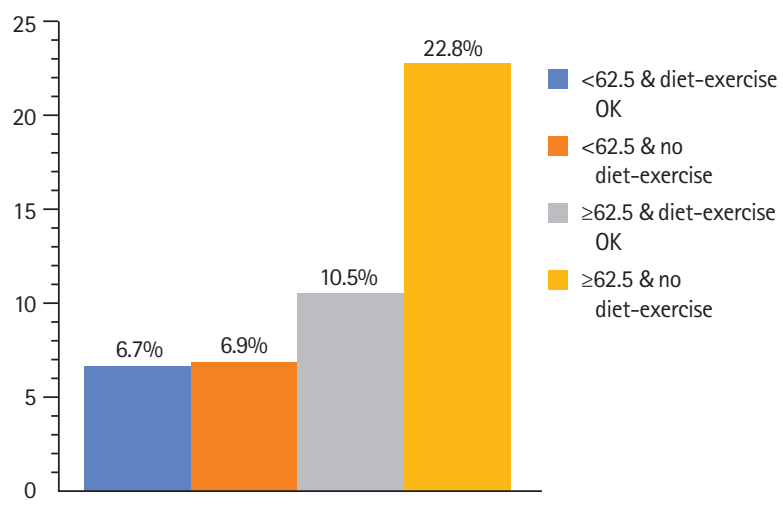

Figure 1. Silent brain infarct (SBI) prevalence according to age and "healthy profile." This figure shows the prevalence of SBI in different groups of patients according to age and the combination of adherence to Mediterranean diet and physical activity.

quency of SBI (22.8\%) (Figure 1).

According to our results, a healthy lifestyle defined by a moderate/high adherence to MeD and a high level of physical activity (>3,000 METS/week) might have a protective effect on SBI prevalence in patients with NVAF. This association with MeD has been previously reported..$^{9}$ Moreover, MeD adherence has been associated with a reduction of cardiovascular events in patients with $\mathrm{AF}$ through an antioxidant effect. ${ }^{10}$ Our study was performed in a population with AF, so as they have an increased risk of stroke, prevention should be more intensive regarding lifestyle. When we considered diet and physical activity as independent variables, we have not found any statistically significant association. This may be explained because people who are more adherent to MeD may be more healthy conscious and indeed more physically active (Supplementary Figure 1). Moreover, this population is thought to smoke less or consume less alcohol, but these confounders were also taken into account." Our study is a single center study in Andalusia, Spain, a region in which $M e D$ is the most frequent type of diet, but our results are consistent with other studies conducted in our country. ${ }^{2}$

\section{Supplementary materials}

Supplementary materials related to this article can be found online at https://doi.org/10.5853/jos.2019.01949.

\section{References}

1. Rosato V, Temple NJ, La Vecchia C, Castellan G, Tavani A, Guercio V. Mediterranean diet and cardiovascular disease: a systematic review and meta-analysis of observational 
studies. Eur J Nutr 2019;58:173-191.

2. Estruch R, Ros E, Salas-Salvadó J, Covas MI, Corella $D$, Arós $F$, et al. Primary prevention of cardiovascular disease with a Mediterranean diet supplemented with extra-virgin olive oil or nuts. N Engl J Med 2018;378:e34.

3. Tsivgoulis G, Psaltopoulou T, Wadley VG, Alexandrov AV, Howard G, Unverzagt FW, et al. Adherence to a Mediterranean diet and prediction of incident stroke. Stroke 2015;46:780-785.

4. Psaltopoulou T, Sergentanis TN, Panagiotakos DB, Sergentanis IN, Kosti R, Scarmeas N. Mediterranean diet, stroke, cognitive impairment, and depression: a meta-analysis. Ann Neurol 2013;74:580-591.

5. O'Donnell MJ, Chin SL, Rangarajan S, Xavier D, Liu L, Zhang $\mathrm{H}$, et al. Global and regional effects of potentially modifiable risk factors associated with acute stroke in 32 countries (INTERSTROKE): a case-control study. Lancet 2016;388:761-775.

6. Craig $C L$, Marshall $A L$, Sjöström $M$, Bauman $A E$, Booth $M L$,

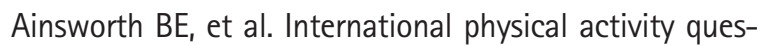
tionnaire: 12-country reliability and validity. Med Sci Sports Exerc 2003;35:1381-1395.

7. Wardlaw JM, Smith EE, Biessels GJ, Cordonnier C, Fazekas $F$, Frayne $R$, et al. Neuroimaging standards for research into small vessel disease and its contribution to ageing and neurodegeneration. Lancet Neurol 2013;12:822-838.

8. González Morales I, Torres Amengual M, Martínez Fernández CA, Luque Vara T. The Mediterranean diet in different age groups. Eur J Health Res 2016;2:73-81.
9. Scarmeas N, Luchsinger JA, Stern Y, Gu Y, He J, DeCarli C, et al. Mediterranean diet and magnetic resonance imaging-assessed cerebrovascular disease. Ann Neurol 2011;69:257268.

10. Pastori D, Carnevale R, Bartimoccia $S$, Nocella $C$, Tanzilli $G$, Cangemi $R$, et al. Does Mediterranean diet reduce cardiovascular events and oxidative stress in atrial fibrillation? Antioxid Redox Signal 2015;23:682-687.

11. Dallongeville J, Marécaux N, Fruchart JC, Amouyel P. Cigarette smoking is associated with unhealthy patterns of nutrient intake: a meta-analysis. J Nutr 1998;128:14501457.

Correspondence: Joan Montaner

Neurovascular Research Group, IBiS/CSIC/University of Seville, Av. Manuel Siurot s/n, 41013, Sevilla, Spain

Tel: +34-955923067

Fax: +34-955923101

E-mail:jmontaner-ibis@us.es

Received: July, 29, 2019

Revised: August, 28, 2019

Accepted: September, 04, 2019

We would like to thank our MRI technicians for their work and Mar Diez from Cajasol for her interest in the project.

The Spanish Ministry of Economy, Industry and Competitiveness (grant RTC-2016 5300-1), the Junta de Andalucia (grant PIN-0144-2016), the European Project ITRIBIS (registration number REGPOT-2013-1) and Cooperative Cerebrovascular Disease Research Network (INVICTUS+, RD16/0019/0015) supported the study. The Fundación Cajasol also contributed to the study.

The authors have no financial conflicts of interest. 
Supplementary Table 1. Baseline characteristics $(n=443)$

\begin{tabular}{|c|c|}
\hline Characteristic & Value \\
\hline Men & $255(57.6)$ \\
\hline Age $(y r)$ & $65 \pm 8$ \\
\hline Hypertension & $227(51.2)$ \\
\hline Diabetes & $25(5.6)$ \\
\hline Hyperlipidemia & $168(37.9)$ \\
\hline \multicolumn{2}{|l|}{ Tobacco } \\
\hline Non-smoker & $371(83.7)$ \\
\hline Smoker & $60(13.5)$ \\
\hline Ex & $12(2.7)$ \\
\hline Alcohol (>78 g/wk) & $108(24.4)$ \\
\hline Vascular disease (myocardial infarction, complex aortic plaque and peripheral artery disease) & $11(2.5)$ \\
\hline Congestive heart failure & $5(1.1)$ \\
\hline Renal insufficiency & $6(1.4)$ \\
\hline \multicolumn{2}{|l|}{ Atrial fibrillation type } \\
\hline Paroxysmal & $366(83)$ \\
\hline Persistent & $27(6.1)$ \\
\hline Permanent & $48(10.9)$ \\
\hline Antiplatelet therapy & $330(74.5)$ \\
\hline Antiarrhythmics & $242(54.6)$ \\
\hline \multicolumn{2}{|l|}{ Mediterranean diet adherence } \\
\hline Very low & $26(5.9)$ \\
\hline Low & $119(26.9)$ \\
\hline Moderate & $258(58.2)$ \\
\hline High & $40(9)$ \\
\hline \multicolumn{2}{|l|}{ Physical activity } \\
\hline Low & $121(27.3)$ \\
\hline Moderate & $156(35.2)$ \\
\hline High & $166(37.5)$ \\
\hline Healthy profile & $121(27.3)$ \\
\hline
\end{tabular}

Values are presented as number (\%) or mean \pm standard deviation. 
Supplementary Table 2. Univariate analysis

\begin{tabular}{|c|c|c|c|}
\hline Varible & SBI $(+)(n=66,14.9 \%)$ & SBI $(-)(n=377,85.1 \%)$ & $P$ \\
\hline Men & $40(60.6)$ & $215(57.0)$ & 0.588 \\
\hline Age (yr) & $71 \pm 7$ & $65 \pm 8$ & $<0.001$ \\
\hline Hypertension & $32(48.5)$ & $195(51.7)$ & 0.627 \\
\hline Diabetes & $5(7.6)$ & $20(5.3)$ & 0.461 \\
\hline Hyperlipidemia & $28(42.4)$ & $140(37.1)$ & 0.414 \\
\hline Tobacco & & & 0.416 \\
\hline Non-smoker & $52(78.8)$ & $319(84.6)$ & \\
\hline Smoker & 11 (16.7) & 49 (13.0) & \\
\hline Ex & $3(4.5)$ & $9(2.4)$ & \\
\hline Alcohol (>78 g/wk) & $24(36.4)$ & $84(22.3)$ & 0.014 \\
\hline Vascular disease & $2(3)$ & $9(2.4)$ & 0.757 \\
\hline Congestive heart failure & $1(1.5)$ & $4(1.1)$ & 0.747 \\
\hline Renal insufficiency & $2(3)$ & $4(1.1)$ & 0.202 \\
\hline Chronic liver disease & $0(0)$ & $1(0.3)$ & 0.675 \\
\hline \multicolumn{4}{|l|}{ Atrial fibrillation type } \\
\hline Paroxysmal & $50(75.8)$ & 316 (83.8) & 0.208 \\
\hline Persistent & $5(7.6)$ & $22(5.8)$ & \\
\hline Permanent & $11(16.7)$ & $37(9.8)$ & \\
\hline Antiplatelet therapy & $52(78.8)$ & $278(73.7)$ & 0.385 \\
\hline Antiarrhythmics & $34(51.5)$ & $208(55.2)$ & 0.582 \\
\hline Statins & $27(40.9)$ & $113(30)$ & 0.078 \\
\hline Mediterranean diet adherence & & & 0.614 \\
\hline Very low & $3(4.5)$ & $23(6.1)$ & \\
\hline Low & $22(33.3)$ & $97(25.7)$ & \\
\hline Moderate & $36(54.5)$ & $222(58.9)$ & \\
\hline High & $5(7.6)$ & $35(9.3)$ & \\
\hline Physical activity & & & 0.106 \\
\hline Low & $25(37.9)$ & $96(25.5)$ & \\
\hline Moderate & $21(31.8)$ & 135 (35.8) & \\
\hline High & $20(30.3)$ & $146(38.7)$ & \\
\hline Healthy profile & $11(16.6)$ & $110(30)$ & 0.035 \\
\hline
\end{tabular}

Values are presented as number (\%) or mean \pm standard deviation.

$\mathrm{SBI}$, silent brain infarct. 


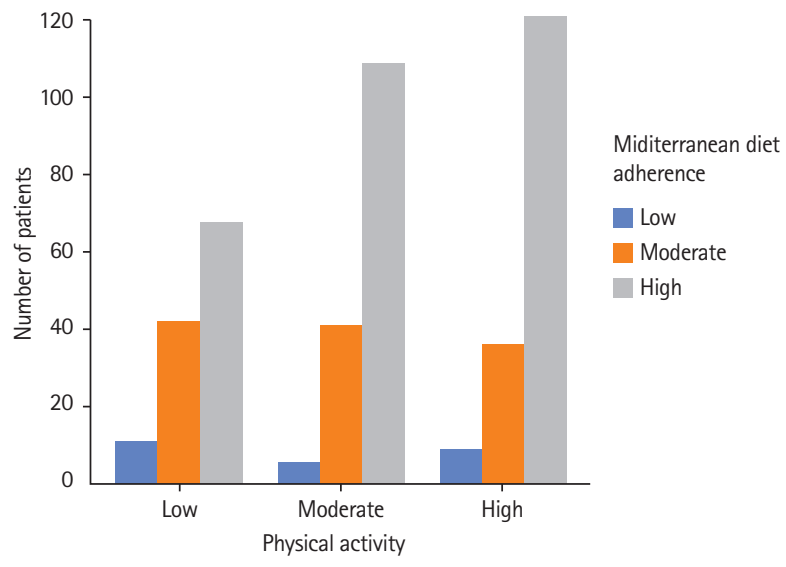

Supplementary Figure 1. Graphic showing distribution of patients regarding adherence to Mediterranean diet and physical activity. 
